\title{
Lacrimal gland adenoid cystic carcinoma: report of an unusual case with literature review
}

\author{
Kenza Benali $i^{1,2}$, Houda Benmessaoud $d^{1,2}$, Jihan Aarab ${ }^{1,2}$, Abdelati Nourreddine ${ }^{1,3}$, Hanan El Kacemi ${ }^{1,2}$, \\ Sanaa El Majjaoui ${ }^{1,2}$, Tayeb Kebdani ${ }^{1,2}$, Noureddine Benjaafar ${ }^{1,2}$ \\ ${ }^{1}$ Department of Radiation Oncology, National Institute of Oncology, Rabat, Morocco \\ ${ }^{2}$ Faculty of Medicine, Mohammed V University, Rabat, Morocco \\ ${ }^{3}$ Équipe de Science de la Matière et du Rayonnement, Department of Physics, Mohammed V University, Faculty of Science, Rabat, Morocco
}

Received: February 2, 2021

Revised: March 21, 2021

Accepted: March 29, 2021

\section{Correspondence:}

Kenza Benali

Department of Radiation Oncology,

National Institute of Oncology,

Avenue Allal El Fassi, 10000, Rabat,

Morocco

Tel: +212673579020

Fax: +212537712455

E-mail: benali.kenza.991@gmail.com

ORCID:

https://orcid.org/0000-0001-9321-9252
Lacrimal gland adenoid cystic carcinomas are rare, aggressive orbital tumors characterized by poor overall prognosis, tendency for local recurrence and metastasis despite aggressive treatment. Treatment continues to be controversial. Many authorities today will often initiate surgery (orbital exenteration with or without bone removal vs. globe-sparing resection) and adjuvant radiotherapy (external beam or proton beam therapy). We introduce a case of lacrimal gland adenoid cystic carcinoma treated with orbital exenteration and adjuvant volumetric modulated arc therapy, and discuss the related literature.

Keywords: Adenoid cystic carcinoma, Lacrimal gland, Orbital exenteration, Adjuvant radiotherapy, Volumetric arc therapy

\section{Introduction}

Adenoid cystic carcinoma (ACC) is a rare malignancy of the secretory glands characterized by slow growth kinetics, prolonged clinical course and perineural invasion. ACC was first described in literature by Theodore Billroth, and was initially named "cylindroma" because of its specific histopathologic characteristics [1]. According to the Surveillance, Epidemiology, and End Results (SEER) database, ACC rising in the eye and orbit, including the lacrimal gland, lacrimal sac, and nasolacrimal duct, accounted for only 1.8\% of total patients. The lacrimal gland was most commonly involved in over $80 \%$. ACC is the most common malignant histology of the lacrimal gland, accounting for $25 \%-40 \%$ of all epithelial tumors of the lacrimal gland [2].
Lacrimal gland ACC (LGACC) is associated with poor long-term disease-free survival (DFS) profile that is complicated by complex orbital anatomy and aggressive behavior, high recurrence rates and significant morbidity and mortality. In the literature, the overall tumor-related mortality of LGACC is $10 \%-87 \%$, with survival rates of less than $50 \%$ at 5 years and $20 \%$ at 10 years regardless of the local treatment regimens. Perineural invasion is considered an indicator of poor prognosis, because of the inherent risks of spread to the skull base and local recurrence. ACC can metastasize via haematogenous spread to lungs, brain and bone in decreasing order of frequency [3].

Defining a single best treatment strategy has been difficult because of the rarity of lacrimal ACC. The appropriate local therapy for LGACC is a subject of controversy. Some authors advocate con- 
servative surgery followed by external beam radiation therapy (EBRT) or proton beam therapy, whereas others believe that radical surgery probably results in better local control and possibly better long-term survival [4].

This case report provides insight to an individualized treatment approach in a patient with ACC of the left lacrimal gland referring to our institution, and discusses current recommendations from the literature, with a focus on the role of adjuvant radiotherapy.

\section{Case Report}

A 45-year-old white man, with a 10 pack-year smoking history, presented to the ophthalmology division of Rabat Hospital with a 9-month history of left-sided painful exophthalmos, red eye and diplopia. On examination, left-sided proptosis and restricted eye motility was noted. The patient's visual acuity was 20/20, and visual fields were full. Sensation in the V1 and V2 distribution was intact, and he was without facial or cervical lymphadenopathy.

Contrast-enhanced computed tomography (CT) scan of the head and orbits showed a left intra-orbital expansive lesion, characterized by a strong contrast enhancement, pushing back the globe with grade 3 exophthalmos, invading the optic nerve and the orbital canal, without endocranial damage. The image morphology was more in favor of lymphoma. The magnetic resonance imaging (MRI) scan of the head and orbits demonstrated a $4.1 \times 1.7 \times 2.6-\mathrm{cm}$ left lacrimal gland tumor, infiltrating the superior and lateral rectus muscles, filling the extra-conical fat, encompassing the eyeball without scleral lysis, with osseous destruction of the orbital roof and meningeal enhancement. The tumor invaded the optic nerve over more than $180^{\circ}$, continuing through the optic canal and the lower orbital fissure (Fig. 1).

A biopsy of this mass revealed ACC with perineural invasion. Neck, chest, abdomen and pelvis CT scans ruled out metastatic disease. Clinical stage was cT4cNOMO, according to the American Join Committee on Cancer staging manual (AJCC 8th edition).

Multidisciplinary head and neck tumor board considered that total orbital exenteration would be necessary to achieve optimal disease control, given the extensive involvement of the lacrimal gland and orbit with perineural invasion. Ultimately, after considering his options, the patient gave his written informed consent for exenteration of the left eye.

The pathological report of the operative specimen confirmed the diagnosis of ACC, measuring $2 \times 1.6 \times 1.5-\mathrm{cm}$, optic nerve in contact with the tumor, with perineural invasion, extension in the soft tissues and positive surgical margins (R1). Postoperative MRI found an empty orbital lodge with no sign of residual disease.

The patient was sent to radiation oncology department for evaluation and was taken up for adjuvant treatment. Simulation was performed using the CT simulator (SOMATOM Sensation Open; Siemens, Erlangen, Germany). The patient was immobilized in supine position with a thermoplastic mask covering the head and neck with three fixation points. The patient was instructed to keep his eyes in a fixed position, even during radiotherapy. The planning CT images were acquired from the head to the base of mandible with intravenous iodine contrast enhancement and a slice thickness of 3-mm. The CT DICOM images were transferred to the Monaco treatment planning system version 5.11 (Elekta, Stockholm, Sweden) for target delineation. The acquired images were then co-reg-
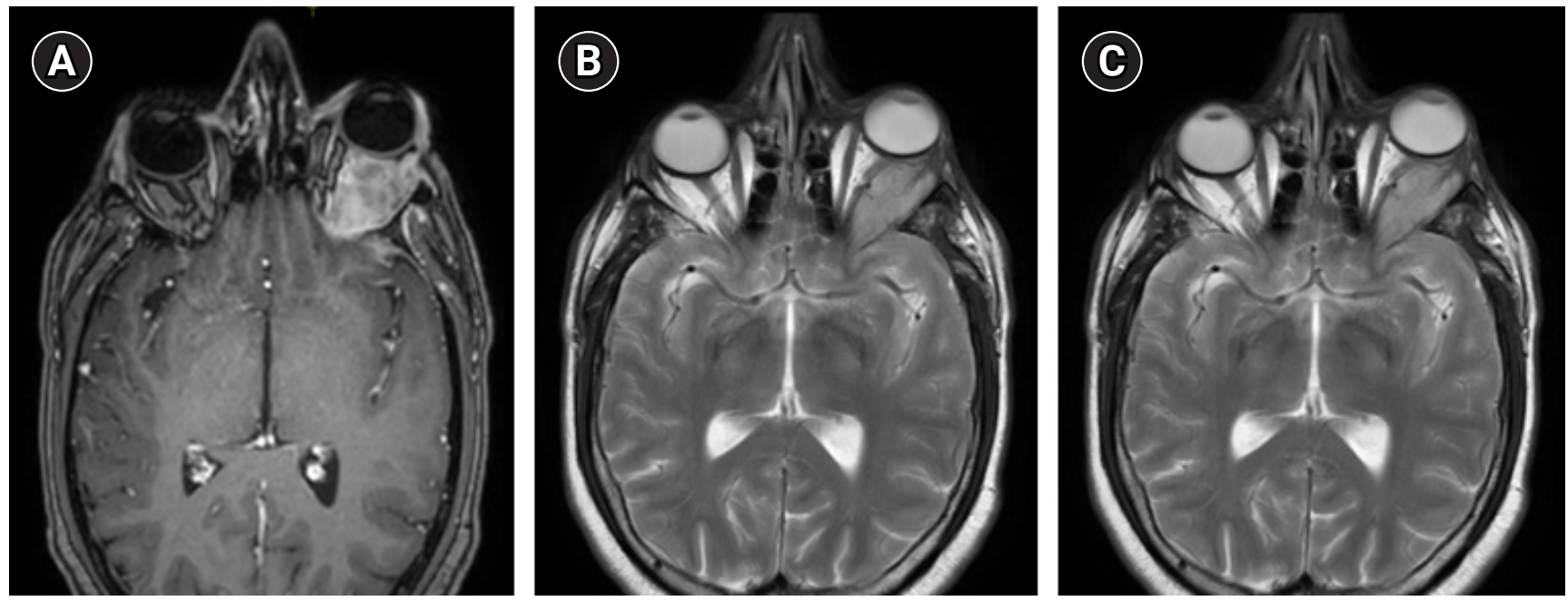

Fig. 1. Preoperative orbital magnetic resonance imaging (MRI). (A) Axial T1-weighted Gadolinium enhanced MRI showing heterogeneous contrast enhancing lesion involving the left orbit. $(B, C)$ Axial T2-weighted images showing isointense soft tissue mass in the lateral aspect of the left orbit. 
istered with diagnostic MRI images to contour the gross tumor volume (GTV) defined before surgery, with the aim of better selecting the clinical target volume (CTV). A 5- $\mathrm{mm}$ isotropic margin was given around GTV to generate high-risk CTV (CTV-HR), and was ad-

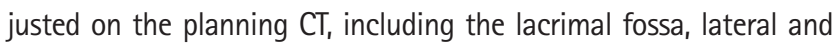
superior portions of the orbit, the entire surgical bed and positive margins to consider the microscopic disease. Due to perineural invasion, this volume comprised also a complex "neuronal" volume, in- cluding: the upper part of the trigeminal ganglion, where the ophthalmic nerve arises; the entire path of the ophthalmic nerve, extending forward along the lateral wall of the cavernous sinus, below the oculomotor and trochlear nerves and through the superior orbital fissure; and the entire path of the lacrimal nerve, running along the upper border of the lateral rectus, parallel to the lacrimal artery and communicating with the zygomatic branch of the maxillary nerve, finally entering the lacrimal gland. The planning target
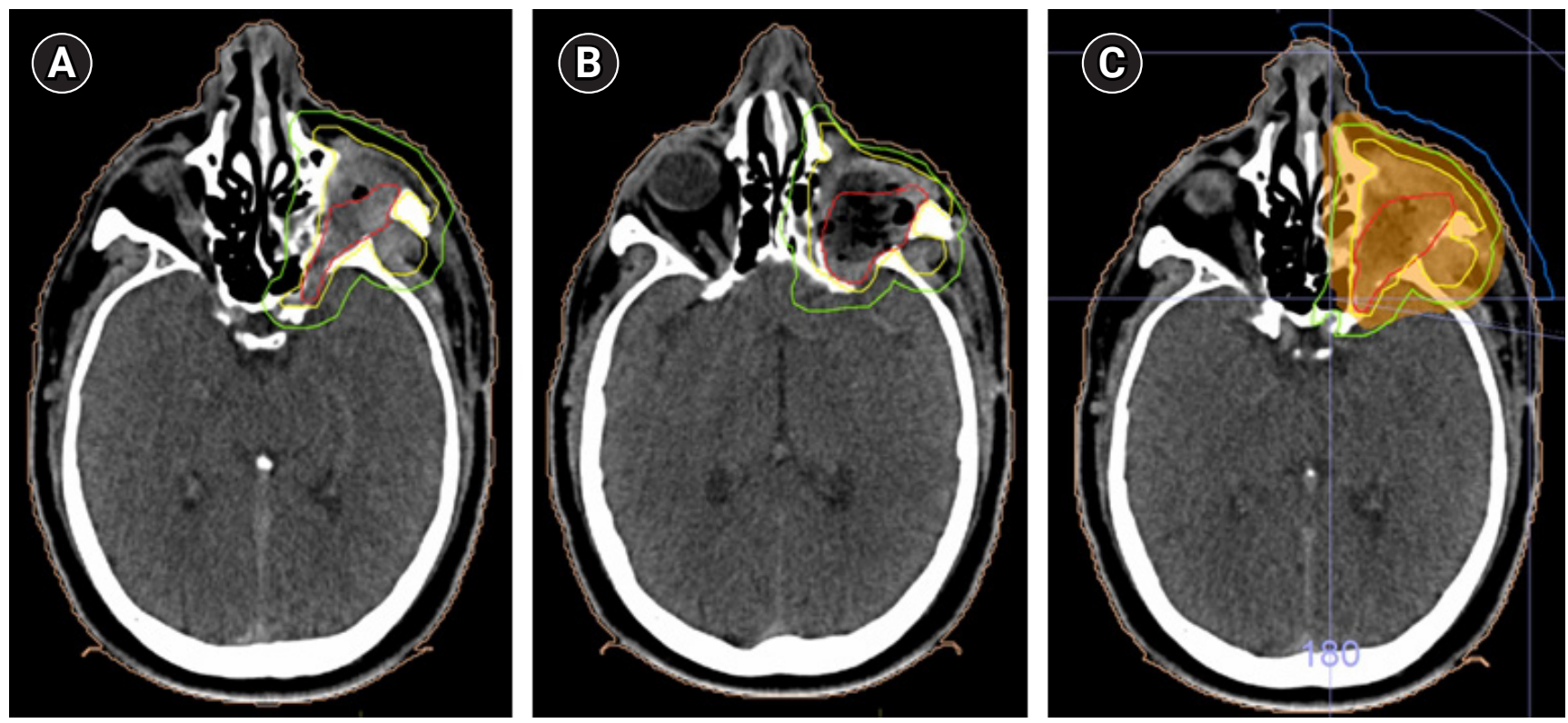

Fig. 2. (A, B) Axial views of the planning computed tomography scan showing the target volumes: preoperative GTV in red, CTV-HR and PTV-HR in yellow and green, respectively. (C) Volumetric modulated arc therapy was delivered via to the region of high risk, using a $0.5-\mathrm{cm}$ thick bolus (in blue), to doses of 66 Gy (95\% isodose line in orange). GTV, gross tumor volume; CTV, clinical target volume; PV, planning target volume; HR, high risk.
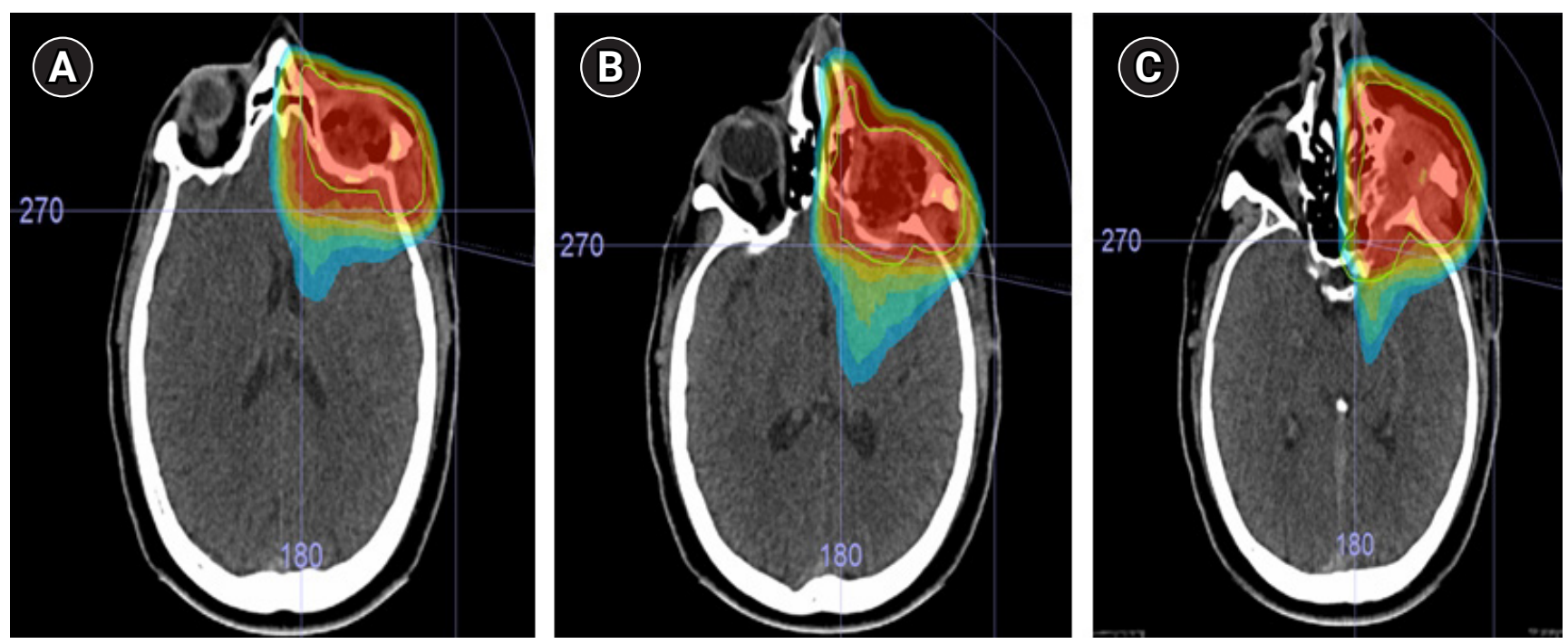

Fig. 3. (A-C) Consecutive axial views from superior to inferior of the planning CT scan with $66 \mathrm{~Gy}$ (100\%; in red) and $62.7 \mathrm{~Gy}$ (95\%; in orange) isodose lines. 
volume (PTV) was created adding a 5-mm expansion around the corresponding CTV, except in the skin direction (Figs. 2, 3). No elective nodal volume was considered. Organs-at-risk (OARs) were contoured according to the Radiation Therapy Oncology Group (RTOG) atlas for normal tissue contouring. OARs consisted of cord, brainstem, optic chiasm, right eye, right lens, right retina, right optic nerve, temporal lobes and cochleae. A dose prescription of $66 \mathrm{~Gy}$ in 2 Gy daily fractions (33 fractions) was given to the PTV to reach the microscopic disease without the limitations placed by an intact globe. Two-arc volumetric modulated arc therapy (VMAT) plan with 6-MV photon beams was set by the medical physicist, using a 0.5$\mathrm{cm}$ thick bolus. Planning goals for the PTV were the following: at least 90\% and 95\% of the prescribed total dose (PTD) encompassing at least 98\% and 95\% of PTV, respectively $\left(\mathrm{V}_{90 \%} \geq 98 \%\right.$ and $V_{95 \%} \geq 95 \%$, respectively); and no more than 2\% of PTV received more than $107 \%$ of the PTD $\left(V_{107} \leq 2 \%\right)$. The following constraints were set for some OARs: for cord, the maximum dose received by $2 \%$ of its volume less than $45 \mathrm{~Gy}\left(\mathrm{D}_{2 \%}<45 \mathrm{~Gy}\right)$; for brainstem, $\mathrm{D}_{2 \%}$ $<55 \mathrm{~Gy}$; for optic chiasm, $\mathrm{D}_{2 \%}<60 \mathrm{~Gy}$; for temporal lobes, $\mathrm{D}_{2 \%}$ $<65 \mathrm{~Gy}$; lastly, the mean dose received by right eye, cochleae and right lens less than 30,45 and $10 \mathrm{~Gy}$, respectively $\left(D_{\text {mean }}<30,45\right.$ and $10 \mathrm{~Gy}$, respectively). We restricted the right optic nerve mean dose to $48 \mathrm{~Gy}$ and a maximum of < $54 \mathrm{~Gy}$. Doses were acceptable for all OARs (Table 1) with homogeneous uniform dose coverage in PTV with 1.08 conformity index. DVH distribution is shown in Fig. 4.

Table 1. Main dosimetric results for organs-at-risk

\begin{tabular}{lcc}
\hline Structure & Parameter & Dosimetric results (Gy) \\
\hline Spinal cord & $D_{\text {max }}$ & 0.8 \\
Brainstem & $D_{2 \%}$ & 45.5 \\
Optic chiasm & $D_{\text {max }}$ & 54.2 \\
& $D_{2 \%}$ & 52.4 \\
Right optic nerve & $D_{\text {max }}$ & 28.8 \\
Left temporal lobe & $D_{2 \%}$ & 66.7 \\
Right temporal lobe & $D_{2 \%}$ & 32.1 \\
Right retina & $D_{\text {max }}$ & 22.1 \\
Right eye & $D_{\text {mean }}$ & 12.2 \\
Left cochleae & $D_{\text {mean }}$ & 22.4 \\
Right cochleae & $D_{\text {mean }}$ & 15.5 \\
Right lens & $D_{\text {max }}$ & 8.7 \\
\hline
\end{tabular}

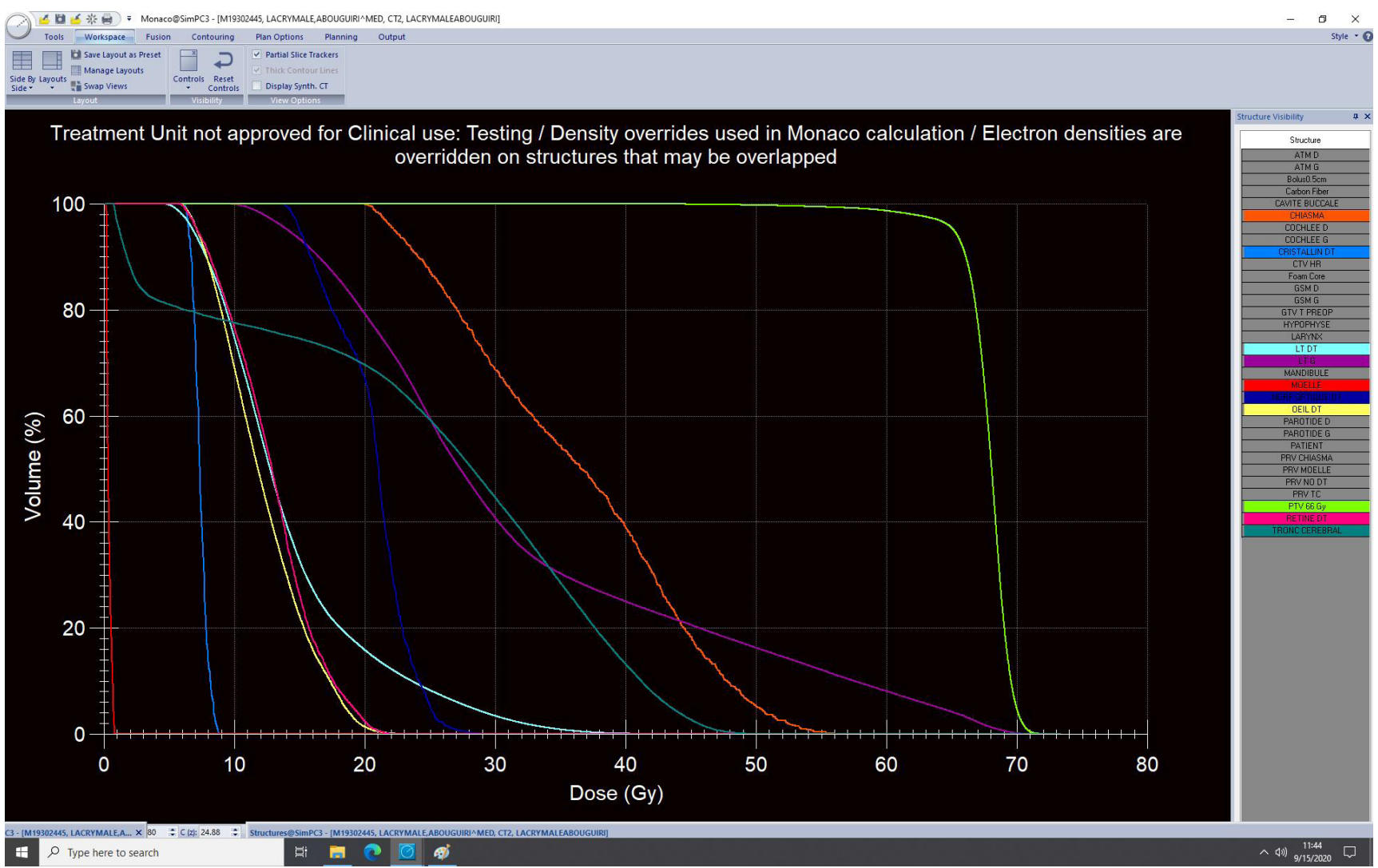

Fig. 4. Dose-volume histogram distribution of the following structures: planning target volume (green), chiasma (orange), left temporal lobe (purple), right temporal lobe (cyan), spinal cord (red), brainstem (dark green), right optic nerve (dark blue), right retina (pink), right lens (blue), and right globe (yellow). 
VMAT plan was delivered using Elekta Versa HD. Patient set-up was verified weekly by $\mathrm{kV}$ cone beam CT imaging prior to treatment. Systemic therapy was not recommended because of the lack of clear supporting evidence for benefit in this setting. During radiotherapy, the patient was assessed for treatment-related toxicity once a week by the radiation oncologist. The patient tolerated the treatment well and experienced reversible grade 1 periorbital dermatitis according to the Common Terminology Criteria for Adverse Events version 5.0 (CTCAE v5.0). No reduction of visual acuity was recorded. There were no treatment interruptions.

After radiotherapy completion, the patient was clinically evaluated every 3-6 months, addressing disease status and late adverse effects. Head and neck MRI and thorax and abdomen CT scans were prescribed on a regular basis every 6 months and 12 months, respectively. After a period of 24-month follow-up, no evidence of local or distant recurrence was found. The patient remains asymptomatic and is still under regular follow-up to date.

\section{Discussion}

ACC is an uncommon malignancy that arises in secretory glands and accounts for about $1 \%$ of all head and neck malignancies. It is the most common primary malignant epithelial tumor of the lacrimal glands and accounts for approximately $1.6 \%$ of all orbital tumors $[2,5]$.

LGACC has a propensity for early perineural invasion, bony and intracranial spread. It is associated with aggressive biology and poor prognosis. Despite aggressive local treatment, LGACC show a high rate of local recurrence and late distant metastasis, with significant resultant mortality [4].

It is mainly an adult tumor with a peak incidence defined in the fourth decade of life, although occurrence is possible at any age [2]. The onset of evolution is usually marked by exophthalmos or ptosis, facial asymmetry owing to displacement of the eyeball and swelling of the lacrimal gland. Pain is a cardinal symptom in patients with LGACC, caused by a growth pattern that leads to early peripheral nerve and extraocular muscle invasion. Lacrimation, reduced eye motility, diplopia and visual changes have also been reported in patients with ACC. The duration of symptoms before first ophthalmic consultation is approximately 6 months. Although, the delay in diagnosis or referral is not unique in our case. Ni et al. [6] have reported that fewer than 15\% of patients were diagnosed within 2 months and $72 \%$ of patients started treatment after 1 year because of delay in diagnosis. Imaging is an important diagnostic tool when establishing a presurgical evaluation of lacrimal gland tumors. MRI is advantageous for early detection of small tumors and for evaluating tumor extension and perineural spread, but it re- mains unable to differentiate between a malignant and benign tumor [5]. The final diagnosis can only be established after pathological evaluation of a biopsy.

Because of the rare nature of $L G A C C$, a randomized clinical trial is not likely. Consequently, guidance on the best possible treatment must rely on retrospective data and small, single institution series. The optimal management for LGACC remains unresolved.

Surgery is usually the first local treatment in LGACC [7]. Taking the correct surgical approach to these lesions is probably one of the most critical management decisions and can save the patient from unnecessary morbidity and mortality. Residual tumor burden following surgery is a significantly important determinant on both progression-free survival and overall survival. Careful attention should to be paid to minimize gross residual disease at surgery [8]. The rarity of lymph node metastasis (4\% to 9\%) suggests that lymph node dissection is unnecessary $[7,9]$.

Worldwide, the extent of surgical resection (orbital exenteration with or without bone excision versus globe-preserving surgery) remains an unsolved issue [10]. Because of a historically poor prognosis, orbital exenteration has been the most common surgery for LGACC in the belief that it improves survival $[3,11]$. However, there is much controversy as to how much radical surgery contributes to the outcome of the patient. In the literature, distant relapse and cancer-related mortality risks are high after orbital exenteration, which highlights the locally invasive and metastatic behavior of this disease [5]. Additionally, comparative case series found that there is no difference in either survival or tumor recurrence for lacrimal gland carcinoma treated with cranio-orbital resection, or eye-preserving tumor excision and radiotherapy $[12,13]$. Moreover, studies have demonstrated that the quality of life of patients who underwent orbital exenteration is markedly reduced because of functional disability and disfigurement [14]. Consequently, eye-preserving surgery followed by adjuvant radiotherapy has recently gained popularity. Data from the literature suggest that eye-sparing surgery with adjuvant radiotherapy can achieve satisfactory results in patients with $\mathrm{T} 1-\mathrm{T} 2$ orbit-confined lacrimal gland carcinoma $[4,15,16]$. However, the risk of local recurrence is increased with conservative surgery in patients with advanced-stage disease (T3T4) at initial diagnosis. Orbital exenteration and adjuvant radiotherapy seems to be a more reasonable option in this group of patients $[10,16,17]$.

In our case, total exenteration was performed after obtaining the informed consent of the patient due to locally advanced disease. However, this surgery can prove challenging given the complex anatomical characteristic of the orbit and the tumor's tendency to spread along nerve tracts. Negative resection margins could not be obtained. 
Adjuvant radiotherapy is recommended after surgical resection, regardless of its extent, especially for patients at high risk of recurrence, including those with an advanced stage tumor and/or multiple unfavorable pathological factors such as bony structure invasion, positive surgical margins, lymphovascular space invasion, or perineural invasion $[10,15,17]$.

Most of the literature available evaluates the use of conventional EBRT. Adjuvant radiation therapy has proven to be effective in preventing locoregional recurrences with local control rates of approximately $50 \%-80 \%$ at 5 years, despite no benefit in survival $[10,18]$. Addition of radiotherapy following incompletely resected ACC (RO/R1) could decrease the risk of progression, but not following $\mathrm{R} 2$ resection $[8,17]$.

Due to the horseshoe-shaped target volume and the proximity to radiosensitive normal structures of lacrimal gland tumors, they might be good candidate for advanced radiotherapy technologies, such as proton beam therapy and intensity-modulated radiation therapy (IMRT) because of their high accuracy and ability to deliver higher radiation doses to the tumor and to spare surrounding tissues. Recently, the introduction of VMAT further improved normal tissue sparing, target coverage and delivery efficiency, compared with conventional IMRT in head-and-neck cancers (HNC) [19]. Very few reports in the literature specifically relates to the use of IMRT or VMAT in LGACC. A case reported by Orlandi et al. [20] presented favorable outcomes in two patients with lacrimal gland cancer treated by postoperative VMAT, with no evidence of disease at 18-month follow-up. To the best of our knowledge, this is the second report describing the use of postoperative VMAT in the management of lacrimal gland malignancies.

In our case, target volumes were selected according to examinations, initial radiological extension of the disease and pathology report. The GTV was accurately outlined on the preoperative MRI and adjusted on the planning CT. Following the international guidelines of the Danish Head and Neck Cancer group (DAHANCA), we defined a CTV-HR including the preoperative GTV plus a 5-mm margin, covering the entire surgical bed and positive margins, along with the lateral orbital wall, superior orbital fissure, orbital roof, and nerve tract up to the skull base. No elective nodal volume was considered because routinely prophylactic nodal irradiation for lacrimal glands malignancies is not recommended [12]. PTV was generated with 5-mm margin for daily setup variation. We prescribed a dose of 66 Gy with standard fractionation to reach the microscopic disease after $\mathrm{R} 1$ resection, which was consistent with data reported in the literature. Tumor control is related to radiation dose and that a dose of at least $60 \mathrm{~Gy}$ is required to obtain an improved control. In a recent review, von Holstein and coworkers [5] reported fractionation schemes for lacrimal gland ACC and non-ACC epithe- lial tumors, suggesting a postoperative dose of 66 Gy for ACC with extracapsular spread, positive or close margins or involved nodes.

No concomitant chemotherapy was delivered in our case as there are still no clear data in the literature in favor of this combined approach [10]. Besides, the role of postoperative chemotherapy for LGACC remains unclear.

After 27 months, our patient is free of recurrent disease despite advanced perineural invasion, locally invasive features and positive surgical margins. He experienced minimal acute toxicity and showed no sign of late toxicity at last follow-up. Our case suggests that postoperative VMAT can potentially offer an improved disease control with moderate acute and late side effects for lacrimal gland tumors in the context of a multidisciplinary therapeutic strategy. However, long-term outcome data are not available yet, and additional evidence is required to further elucidate the efficacy and safety of adjuvant VMAT in LGACC.

In conclusion, LGACC is a rare and slowly progressing disease, associated with poor local control, distant metastasis and significant morbidity and mortality rates. Delayed diagnosis, tendency for peri-neural invasion, infiltration to periosteum and local recurrence are factors resulting in poor outcome. To date, optimal treatment is still debated. First treatment conventionally consists in surgery followed by postoperative radiotherapy. Ongoing discussions focus on the optimal dose, fractionation and technique of radiotherapy. ACC is classically considered as a radioresistant tumor, and dose escalation to the focal region of risk is essential to hope for a curative irradiation. New irradiation techniques such as VMAT proved to be effective and safe in irradiating orbital tumors. The role of systemic therapy is yet to be established. Close follow-up after treatment should be undertaken to evaluate both recurrence and radiation-related complications. Larger multicenter studies can yield additional insight and should be planned in the future.

\section{Conflict of Interest}

No potential conflict of interest relevant to this article was reported.

\section{References}

1. Billroth T. Untersuchungen uber die Entwicklung der Bluttgefasse. Berlin, Germany: Reimer; 1856. p. 5-69.

2. Ellington $\mathrm{CL}$, Goodman $\mathrm{M}$, Kono SA, et al. Adenoid cystic carcinoma of the head and neck: Incidence and survival trends based on 1973-2007 Surveillance, Epidemiology, and End Results data. Cancer 2012;118:4444-51.

3. Esmaeli B, Ahmadi MA, Youssef A, et al. Outcomes in patients 
with adenoid cystic carcinoma of the lacrimal gland. Ophthalmic Plast Reconstr Surg 2004;20:22-6.

4. Woo Kl, Yeom A, Esmaeli B. Management of lacrimal gland carcinoma: lessons from the literature in the past 40 years. Ophthalmic Plast Reconstr Surg 2016;32:1-10.

5. Von Holstein SL, Coupland SE, Briscoe D, Le Tourneau C, Heegaard S. Epithelial tumours of the lacrimal gland: a clinical, histopathological, surgical and oncological survey. Acta Ophthalmol 2013;91:195-206.

6. Ni C, D'Amico DJ, Fan CQ, Kuo PK. Tumours of the lacrimal sac: a clinical-pathological analysis of 82 cases. Int Ophthalmol Clin 1982;22:121-40.

7. Esmaeli B, Golio D, Kies M, DeMonte F. Surgical management of locally advanced adenoid cystic carcinoma of the lacrimal gland. Ophthalmic Plast Reconstr Surg 2006;22:366-70.

8. Noh JM, Lee $E_{1}$ Ahn YC, et al. Clinical significance of post-surgical residual tumor burden and radiation therapy in treating patients with lacrimal adenoid cystic carcinoma. Oncotarget 2016; 7:60639-46.

9. Esmaeli B, Yin VT, Hanna EY, et al. Eye-sparing multidisciplinary approach for the management of lacrimal gland carcinoma. Head Neck 2016;38:1258-62.

10. Yang J, Zhou C, Wang Y, Fan $X$, Jia R. Multimodal therapy in the management of lacrimal gland adenoid cystic carcinoma. BMC Ophthalmol 2019;19:125.

11. Wilson KF, Ward PD, Spector ME, Marentette L. Orbitocranial approach for treatment of adenoid cystic carcinoma of the lacrimal gland. Ann Otol Rhinol Laryngol 2011;120:397-400.

12. Skinner HD, Garden AS, Rosenthal DI, et al. Outcomes of malignant tumors of the lacrimal apparatus: the University of Texas MD
Anderson Cancer Center experience. Cancer 2011;117:2801-10.

13. Rose GE, Gore SK, Plowman NP. Cranio-orbital resection does not appear to improve survival of patients with lacrimal gland carcinoma. Ophthalmic Plast Reconstr Surg 2019;35:77-84.

14. Bonanno A, Esmaeli B, Fingeret MC, Nelson DV, Weber RS. Social challenges of cancer patients with orbitofacial disfigurement. Ophthalmic Plast Reconstr Surg 2010;26:18-22.

15. Han J, Kim YD, Woo KI, Sobti D. Long-term outcomes of eye-sparing surgery for adenoid cystic carcinoma of lacrimal gland. Ophthalmic Plast Reconstr Surg 2018;34:74-8.

16. Ahmad SM, Esmaeli B, Williams M, et al. American Joint Committee on Cancer classification predicts outcome of patients with lacrimal gland adenoid cystic carcinoma. Ophthalmology 2009;116:1210-5.

17. Lin YH, Huang SM, Yap WK, et al. Outcomes in patients with lacrimal gland carcinoma treated with definitive radiotherapy or eye-sparing surgery followed by adjuvant radiotherapy. Radiat Oncol 2020;15:156.

18. Roshan V, Pathy S, Mallick S, Chander S, Sen S, Chawla B. Adjuvant radiotherapy with three-dimensional conformal radiotherapy of lacrimal gland adenoid cystic carcinoma. J Clin Diagn Res 2015:9:XC05-7.

19. Teoh M, Clark CH, Wood K, Whitaker S, Nisbet A. Volumetric modulated arc therapy: a review of current literature and clinical use in practice. Br J Radiol 2011;84:967-96.

20. Orlandi E, Takanen S, Giandini T, et al. Postoperative radiotherapy with volumetric modulated arc therapy of lacrimal gland carcinoma: two case reports and literature review. Future Oncol 2014; 10:2111-20. 